\title{
Food therapy for the Traditional Chinese Medicine (TCM) diagnosis of dampness
}

Volume 2 Issue 2 - 2015

Keywords: food, therapy, dampness, TCM, skin, abdomen, jiao, obesity, diabetes, herbs, diet

\section{Introduction}

Please refer to a previous article for a description of exactly what constitutes the TCM diagnosis of Dampness. ${ }^{1}$ In this article, a description of various food and herbal substances that assist in the alleviation of Dampness conditions will be discussed in reference to those conditions in the skin, chest (upper jiao), abdomen (middle jiao) and pelvic region (lower jiao). In addition, the role that foods play in the condition of obesity will also be addressed.

\section{Discussion}

In the first reference, it was demonstrated that the TCM diagnosis of Dampness in developed countries is mainly due to a hormonal imbalance of estrogen and secondarily due to consumption of refined food products and the processes that manufacture those food products. Also, in the main, it is Dampness Heat that is the prevalent condition. Thus, some form of Inflammation accompanies Dampness, in these cases. Thus fluid retention is also a characteristic of Dampness. First, the conditions will be described and then the foods and herbs that are known to alleviate or exacerbate the relative conditions will be presented.

\section{Conditions}

Obesity: Obesity is described as a condition where the body mass index (BMI) is typically 30 or greater. All persons diagnosed as obese have the condition of Dampness Heat and are experiencing some form of chronic inflammation. Your lifestyle choices are definitely going to have to change! Stop eating out and cook your own foods. Learn how to cook and eat healthily.

The most immediate food therapy (FT) for obesity is severe caloric restriction, i.e., to stop eating so much! Then the first and most important foods to restrict in your diet would be all soy food related products like soybean oil, tofu, soy sauce, tempe, edamame, miso, soy milk. These foods have phytoestrogens in them and contribute to your overall body estrogen content. It is the excess body burden of estrogen that causes the adrenal glands to secret cortisol and stimulates fat deposition. This is a positive feedback mechanism and the only way to break it is to stop the ingestion of these foods. Please go online and search for foods that contain phytoestrogens to see if you are consuming a more than normal amount of them.

The second most important food ingredient to eliminate from your diet is High Fructose Corn Syrup. Un-natural levels of fructose interfere with normal carbohydrate metabolism. ${ }^{2}$ This means that you need to read the labels of the processed foods that you eat. The third food ingredient to eliminate is Olestra, Olean and other un-natural oil substitutes. ${ }^{3,4}$

Upper jiao (chest, Lungs): There is a saying in TCM - "The earth element creates dampness and the metal element stores it." In TCM, The organs associated with the earth element are the Stomach and

\author{
Edward F Block \\ Block Institute For Astrobiological Studies, USA
}

Correspondence: Edward F Block, Block Institute For Astrobiological Studies, 375 Dula Springs Road,Weaverville, North Carolina, USA, Email efblockiv@yahoo.com

Received: December II, 2015 | Published: December 16, 2015

Spleen. The organs associated with the metal element are the Lungs and Large Intestine. When dampness is created by impaired digestion, caused by chronic improper nutrition, it likes to end up in the Lungs and Large Intestine. When dampness moves into the Lungs, the usual symptom is phlegm coming up while coughing. This will occur especially after eating something that is inherently difficult to digest such as a milk shake, other cold dairy products, or greasy foods. Dairy products are well known to be a cause of Dampness in TCM.

Middle jiao (abdomen): Type 2 Diabetes is a condition due to high fatty content in the pancreas and thus is a Dampness Heat condition. The best way to decrease pancreatic fat is severe caloric restriction ${ }^{5}$, just as in obesity. Obesity and type 2 Diabetes seem to go hand-inhand.

Lower jiao (pelvic region): Polycystic ovaries, endometriosis and fibroid tumers in the uterus are Dampness Heat conditions. These conditions are due mostly to an excessive body burden of estrogen. Thus, a restriction of estrogenic foods needs to be the first thing that one should do in order to alleviate these conditions as well as overall body weight reduction.

The Liver and Gall Bladder are also affected by the excess consumption of fatty foods. Jaundice is a Dampness condition of the Liver. $^{6}$ Gall stones are a Damnpess condition of the Gall Bladder. ${ }^{7}$ The only way to truly combat "fatty" liver is to severely restrict fatty food intake. Fatty liver and gall stones seem to go together. Most but not all gallstones are composed mostly of cholesterol and the rest of bilirubin, or a mixture of the 2 .

Skin: Rashes, blotches, cholesterol deposits and skin tags are all Dampness Heat conditions of the skin. They are the result of acute responses to ingested foodstuffs and in the case of cholesterol deposits and skin tags, the chronic response to Dampness Heat in the skin. The skin is the largest organ of the body. What the body is not able to eliminate in the faecal waste is absorbed and metabolized by the Liver. Whatever the liver metabolizes ends up in the blood or the bile but may also be stored in the liver. The blood moves all metabolites into 
the rest of the bodily fluids. Some metabolites end up in the adipose tissue (fat cells). But some of the compounds in the blood seem to migrate from the blood into the interstitial fluid of the skin and cause acute Dampness Heat signs and symptoms. These compounds are mostly allergens and involve the immune system. Skin tags seem to be more related to toxic Dampness Heat. Seborrheic keratoses as chronic damp-heat in the skin seem related to a chronic high body burden of estrogens, like skin tags also are.

\section{Foods and herbs for therapy}

\section{Foods and herbs that cause dampness conditions}

Milk products (except yogurt), sugar and sweets, white wheat flour, refined starch and highly processed starch products, excess raw fruits (sugars) and vegetables (mold from the soil), excess mushrooms and fungi, any of the pepper family vegetables, icy and cold beverages (which immobilize immune cells on the walls of digestive track), an excess of fermented foods, yeast containing foods and an excess of vinegar. You may go online and search for other Dampness contributing foods and herbs such as Oranges and Orange juice.

\section{Foods and spices that are known to cause dampness heat}

Drinking alcoholic beverages in any of it's many forms; fatty, greasy and deep fried foods of any kind.

\section{Foods and spices known to relieve dampness \& dampness heat}

Eat more spices that contain yeast/fungus - inhibiting properties such as garlic, onion, cinnamon, ginger, scallion, basil, rosemary, dill, oregano, sage, parsley, cardamom, nutmeg, fennel, anise, clove, coriander, leek, chives. And eat other Dampness relieving foods like Job's Tears (yi yi ren), Euryale (Fox nut) seed (qian shi), Aduki beans (chi xiao dou), Ginkgo nut (bai guo), rye, celery, lettuce, alfalfa, turnips, raw honey and corn. Search online for suitable recipes to combine the above foods and herbs into delicious stir-fry, soup or stew.

Turmeric is a spice that is very anti-inflammatory, anti-dampness and is known to suppress the growth of new blood vessels necessary for the rapid growth of cancerous cells. Turmeric by itself is not very well absorbed by the small intestinal mucosa. However, if you mix turmeric with black pepper, ${ }^{8}$ the absorption increases by 1000 times better! Combine turmeric powder with equal amounts of fresly ground black pepper in olive oil and add to a stir-fry, soup or stew.

\section{Decoction therapy}

One of the best methods for consuming herbal substances is in a decoction like that of tea or coffee. A decoction that the author likes for Dampness Heat in the Lower Jiao is Decaf with Pumpkin Pie Spice (Cinnamon, Clove, Ginger, Allspice, Nutmeg). This is the recipe: Boil water enough for 24 ounces. To a 24 ounce Mason jar add 1 ounce of Maple syrup. Then add 1 teaspoon of Pumpkin Pie Spice to the jar on top of the Maple syrup. Add 2 tablespoons of 100\% Arabica Decafe (decafinated by using the supercritical Carbon Dioxide method, the Ethyl-Acetate method) to an un-bleached or bamboo paper filter. Pour the boiling water over the coffee grounds until the jar is filled to the neck of the jar and remove the filter apparatus. Attach the jar lid and skate for a short time to mix the contents. Let cool and drink from the jar or pour into a suitable container. Enjoy!

For Toxic Dampness in the skin, use 5 Spice Seasoning (Cinnamon, Anise, Ginger, Nutmeg, Clove) instead of Pumpkin Pie Spice with your Decafe. Or you may make a tea with 8 -Star Anise with your green tea (Camellia sinensis) leaves. Gently boil your green tea leaves with 5-6 pieces of 8-Star Anise for 30minutes, let cool and imbibe. Again, use Maple Syrup for sweetener.

You may also make a tea from Italian Herbs (Rosemary, Thyme, Oregano) using Maple Syrup for sweetener. This works well for Dampness Heat in the Middle Jiao. Use 1 tablespoon for 24 ounces of water. Gently boil for 30 minutes.

For general Dampness conditions, making your tea (Camellia sinensis) with Roselle (Hibiscus sabdariffa) ${ }^{9}$ is a delightful combination to both cool you and to alleviate your Dampness condition. Use any sweetener of your choice but not refined sugar please! Refined sugar contributes to Dampness, depletes Calcium from tooth sockets and contributes to osteoporosis by robbing Calcium from bone. Roselle is also a good source of Vitamin C. In addition, Roselle and Yellow Chrysanthemum (Ju Hua) make a good decoction for Dampness Heat in the lower Jiao. Yellow Chrysanthemum is very good for all Liver and Gall Bladder problems. When added to Roselle, they make an excellent anti-Dampness decoction. Use 3-6grams of Ju Hua and the same of Roselle. Each person will need to determine the correct amount of Rosselle to add to the mix as too little does no good and too much has the tendency to constipate. You will need to be in between these 2 extremes. The author likes to add tangerine peel (Chen Pi) to the mix also, 3 grams is sufficient. All goes into 2 quart of gently boiling water for 30 minutes. Again, use the sweetener of your choice but please not refined sugar!

\section{Conclusion}

This article has given the description of Dampness \& Dampness Heat in the case of obesity, the 3 Jiaos and the skin. Some foods, herbs and spices were introduced that contribute and alleviate the Dampness and Dampness Heat diagnoses. Lastly, decoctions used to treat Dampness conditions were presented and how to make them for use was described.

\section{Acknowledgments}

None.

\section{Conflicts of interest}

None.

\section{Funding}

None.

\section{References}

1. Edward FB. The Traditional Chinese Medicine Concept of Dampness Explained In Term Of Modern Medicine. Int $J$ Complement Alt Med. 2015;2(1):00041.

2. http://drhyman.com/blog/2011/05/13/5-reasons-high-fructose-cornsyrup-will-kill-you/

3. http://www.cspinet.org/new/flaynal.html

4. http://www.shape.com/healthy-eating/diet-tips/9-common-foodscontain-toxic-ingredients/slide/4

5. http://www.sciencealert.com/losing-just-1-gram-of-fat-in-the-pancreascan-reverse-type-2-diabetes

6. https://en.wikipedia.org/wiki/Jaundice

7. https://en.wikipedia.org/wiki/Gallstone

8. http://www.turmericforhealth.com/turmeric-benefits/health-benefits-ofblack-pepper-and-turmeric

9. https://en.wikipedia.org/wiki/Roselle (plant) 